\title{
Effect of Posterior Tibial Slope Change on Postoperative Range of Motion and Clinical Outcomes after Posterior Cruciate-Substituting Total Knee Arthroplasty
}

\author{
O-Sung Lee, MD ${ }^{1}$ Jangyun Lee ${ }^{2}$ Myung Chul Lee, MD, PhD 3 Hyuk-Soo Han, MD, PhD ${ }^{3}$ (1)
}

${ }^{1}$ Department of Orthopedic Surgery, Eulji University School of Medicine, Uijeongbu-si, Korea (the Republic of)

2 Department of Orthopedic Surgery, National Medical Center, Seoul, Korea (the Republic of)

${ }^{3}$ Department of Orthopedic Surgery, Seoul National University Hospital, Seoul, Korea (the Republic of)

J Knee Surg 2023;36:87-94.
Address for correspondence Hyuk-Soo Han, MD, PhD, Department of Orthopedic Surgery, Seoul National University Hospital, 101 Daehakro, Jongno-gu, Seoul 110-744, Republic of Korea (e-mail: oshawks7@snu.ac.kr).

\begin{abstract}
Keywords

- total knee arthroplasty

- posterior tibial slope

- range of motion

- clinical outcome

The posterior tibial slope (PTS) is usually adjusted by less than 5 degrees, without considering its individual difference, during posterior cruciate-substituting (PS) total knee arthroplasty (TKA). The effect of these individual changes of PTS would be important because clinical results depending on postoperative PTS were reported conflictingly. We investigated the effect of the change in PTS on the postoperative range of motion (ROM) and clinical scores after PS TKA. We retrospectively reviewed 164 knees from 107 patients who underwent PS TKA with a 2-year follow-up. We analyzed the preoperative and postoperative PTS, ROM, visual analog scale pain scale, Western Ontario and McMaster University Index (WOMAC), Hospital for Special Surgery Knee Score, Knee Society Score, and Forgotten Joint Score (FJS). The association of the absolute change in PTS with ROM and clinical scores was analyzed using correlation analysis and multiple regression analysis. As a result, the mean PTS and mean ROM changed from $9.6 \pm 3.4$ and $120.1 \pm 15.4$ degrees preoperatively to $2.0 \pm 1.3$ and $128.4 \pm 9.3$ degrees postoperatively, and the mean PTS change was $7.6 \pm 3.5$ degrees. The PTS change had no statistically significant association with the postoperative ROM and clinical scoring systems, although it did have a weak positive correlation with WOMAC function, No 10 (difficulty in rising from sitting) (correlation coefficient $=0.342, p=0.041$ ), and moderate positive correlation with the FJS, No. 6 (awareness when climbing stairs) (correlation coefficient $=0.470, p=0.001$ ). The authors concluded that the amount of change in PTS did not affect the postoperative ROM and clinical scores, although proximal tibial resection with a constant target of PTS resulted in individually different changes in the PTS after PS TKA,
\end{abstract}

Patient satisfaction after total knee arthroplasty (TKA) is known to be related to postoperative range of motion (ROM), in that increased ROM improves postoperative function and satisfaction. ${ }^{1,2}$ Among several factors affecting postoperative
ROM, such as sex, age, preoperative ROM, body mass index (BMI), prosthesis design, and surgical techniques, some authors reported that the posterior tibial slope (PTS) correlated with postoperative ROM after TKA. ${ }^{3-6}$ The PTS is the received

December 27, 2020

accepted after revision

April 1, 2021

article published online

May 15, 2021 (c) 2021. Thieme. All rights reserved.

Thieme Medical Publishers, Inc., 333 Seventh Avenue, 18th Floor, New York, NY 10001, USA
DOI https://doi.org/ 10.1055/s-0041-1729967. ISSN 1538-8506. 


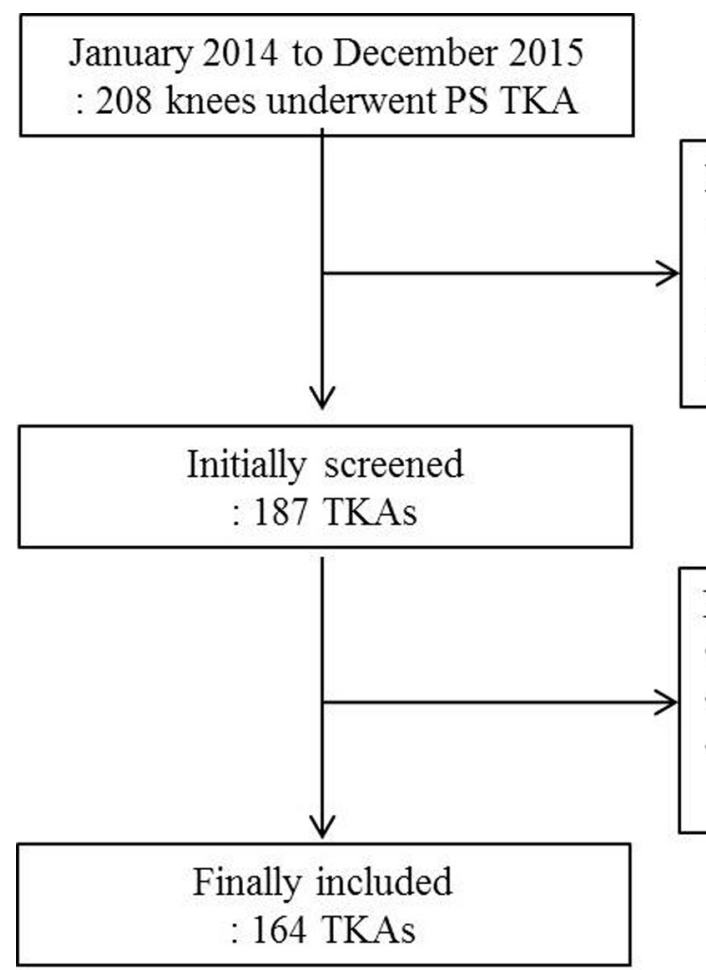

Exclusion: 21

- Other than primary osteoarthritis: 7

- Severe deformity: 4

- Flexion contracture $>20^{\circ}: 4$

- Previous surgery: 6

Exclusion: 23

- Follow up loss: 9

- Incomplete clinical scoring: 11

- Imperfect lateral radiograph for analysis: 3

\section{Finally included \\ 164 TKAs}

Fig. 1 Flowchart of patient inclusion and the reasons for exclusion. PS TKA, posterior cruciate-substituting total knee arthroplasty.

posterior inclination in the geometry of the tibial plateau, and affects the kinematics of the knee joint. ${ }^{7-11}$ Traditionally, it has been reported that an increased PTS leads to better flexion after TKA.,4,6 Therefore, many manufacturers designed the total knee prosthesis with a built-in PTS plus the angle of the postoperative tibial resection. However, there are many concerns surrounding an increased PTS following posterior cruciate-substituting (PS) TKA. Indeed, some studies have shown that the increased PTS in PS TKA does not help maximal flexion, ${ }^{12,13}$ and a large PTS could increase the risk of wear of the polyethylene insert, and result in cam-post impingement. ${ }^{13,14}$ Additionally, an excessive PTS after PS TKA should be avoided to prevent anteroposterior instability of the knee joint, although an increased PTS contributes to a decrease in the maximum quadriceps force and patellofemoral contact force during knee extension. ${ }^{15}$ Moreover, it is generally thought to be better to reduce the PTS to control the flexion gap, since the flexion gap increases more than the extension gap after posterior cruciate ligament (PCL) resection during PS TKA. ${ }^{16}$

de Boer et al reported a PTS of 8.4 degrees in 105 human cadavers. ${ }^{17}$ Despite such variations between individuals, most surgeons adjust the PTS by less than 5 degrees during PS TKA for the reasons described above. ${ }^{15,16,18}$ Therefore, the individual amount of change in PTS varies depending on the preoperative PTS of the affected knee joint. However, little is known about the effect of these individual changes in PTS.

The main purpose of this study was to investigate the effect of individual changes in PTS on ROM and clinical outcomes after PS TKA. We hypothesized that individual change in PTS will have an individually different impact on postoperative ROM or clinical outcomes after PS TKA.

\section{Materials and Methods}

Patients who underwent primary PS TKA from January 2014 to December 2015 were retrospectively reviewed. Only patients with osteoarthritis of the knee joint and who completed a clinical and radiologic evaluation at the 2-year postoperative follow-upwere included. The exclusion criteria were as follows: (1) Arthritis, such as inflammatory or posttraumatic arthritis, other than primary osteoarthritis of the knee joint; (2) bone graft due to severe deformity or bone defect; (3) severe flexion contracture over 20 degrees; and (4) previous open surgery on the affected knee joint. A total of 141 consecutive patients (208 knees) underwent primary PS TKA; however, 38 patients were excluded due to the selection criteria and inadequate follow-up. Finally, a total of 164 TKAs in 107 patients were available for radiologic and clinical evaluation preoperatively and 2 years postoperatively (-Fig. 1). The demographics of the included patients are shown in - Table 1 . The study was approved by the Institutional Review Board of our hospital.

Table 1 Demographics

\begin{tabular}{|l|l|}
\hline Number of knees & 164 \\
\hline Mean age $(\mathrm{y})^{\mathrm{a}}$ & $70.3 \pm 6.1$ \\
\hline Gender (male/female) & $10 / 154$ \\
\hline Laterality (right/left) & $80 / 84$ \\
\hline Body mass index $\left(\mathrm{kg} / \mathrm{m}^{2}\right)^{\mathrm{a}}$ & $27.2 \pm 3.5$ \\
\hline
\end{tabular}

${ }^{a}$ Values are presented as the mean and the standard deviation. 


\section{Surgical Technique and Postoperative Rehabilitation}

All TKAs were performed by a single surgeon using a primary fixed bearing PS type TKA system (Attune; DePuy Synthes, Warsaw, IN), of which a built-in tibial slope was set at 0 degree and recommended to set the postoperative PTS at 3 degrees. Medial parapatellar arthrotomy was performed after a midline skin incision, and both cruciate ligaments were sacrificed before bone cutting. Resection of the distal femoral condyle was conducted according to the lateral distal femoral angle, following which proximal tibial cutting was performed. The thickness of tibial resection was based $\sim 10 \mathrm{~mm}$ referenced off the lateral tibial plateau. The resection was perpendicular to the tibial mechanical axis on the coronal plane, with a target PTS of 0 to 3 degrees using an extramedullary guide. The anterior surface and sagittal axis of the tibia that is visually verified were used as references. The extension gap was checked using the knee balancer (Stryker Howmedica Osteonics, Allendale, NJ) and spacer block. ${ }^{19}$ The size and rotational alignment of the femoral component were determined by the modified gap technique using manual distraction to set the knee at 90 degrees of flexion, and anteroposterior cutting of the femur was performed parallel to the tibial cutting plane. ${ }^{20}$ And, flexion gap was also checked using the knee balancer and spacer block. The optimal sizing and positioning of the tibial tray were decided using a reference with the margin of the tibia tray aligned with the lateral tibial plateau and anterior tibial cortical line. The thickness of the polyethylene insert was determined after trying the trial insert.

With regard to the postoperative rehabilitation protocol, patients began knee flexion exercises using a continuous passive motion exercise machine on the first day after surgery. Patients were allowed to perform straight leg raising exercises immediately, while in the recovery room, and were allowed to stand or walk using a knee immobilizer for support and a walker for balance on the first day after surgery.

\section{Radiologic and Clinical Evaluations}

Radiologic and clinical evaluations were performed preoperatively and annually after surgery. Data of all cases at the 2year postoperative follow-up were collected, and preoperative medical records and radiographs were reviewed retrospectively. The preoperative and postoperative PTSs were measured on a true lateral radiograph of the knee joint. True lateral radiograph of the knee joint means that the posterior aspects of the medial and lateral femoral condyles are superimposed and the patellofemoral joint is open and projected free. Additionally, there is slight superimposition of the fibular head with the tibia. ${ }^{21,22}$ A horizontal line (A) was drawn perpendicular to a line connecting the midpoints of the outer cortical diameter at 5 and $15 \mathrm{~cm}$ distal to the knee joint. A line was drawn parallel to the medial tibia plateau of the proximal tibia (B). ${ }^{22}$ The angle formed by lines A and $\mathrm{B}$ represents the PTS (-Fig. 2). The patellar height was measured according to the Insall-Salvati ratio and BlackburnePeel ratio. ${ }^{23,24}$ The Insall-Salvati ratio $(a / b)$ is defined as the length of the patellar tendon (a) divided by the diagonal

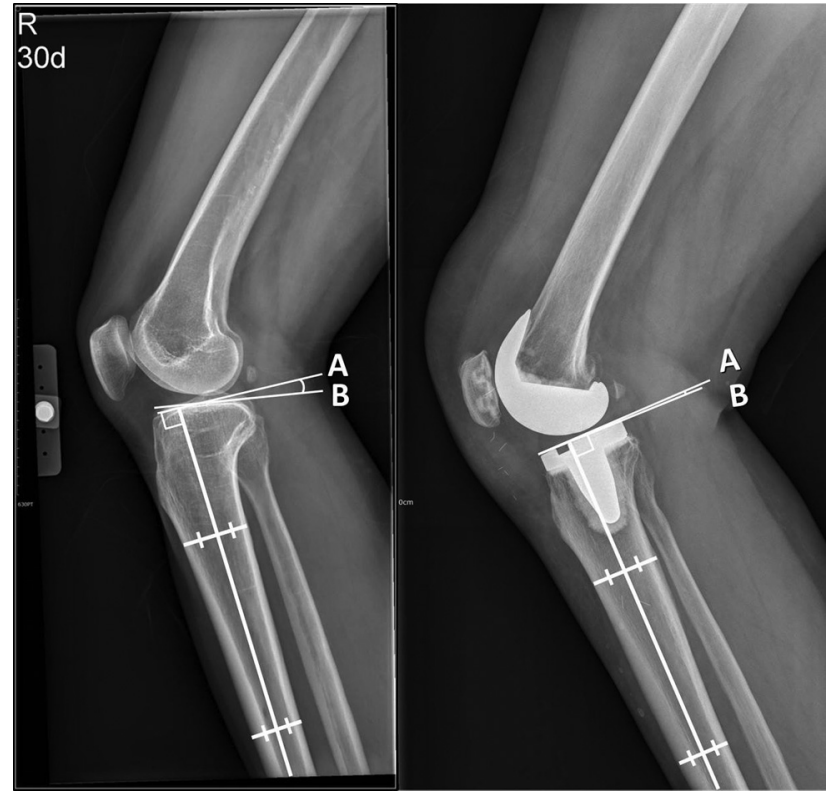

Fig. 2 Measurement of the posterior tibial slope on preoperative and postoperative radiographs (angle formed by lines $A$ and $B$ ).

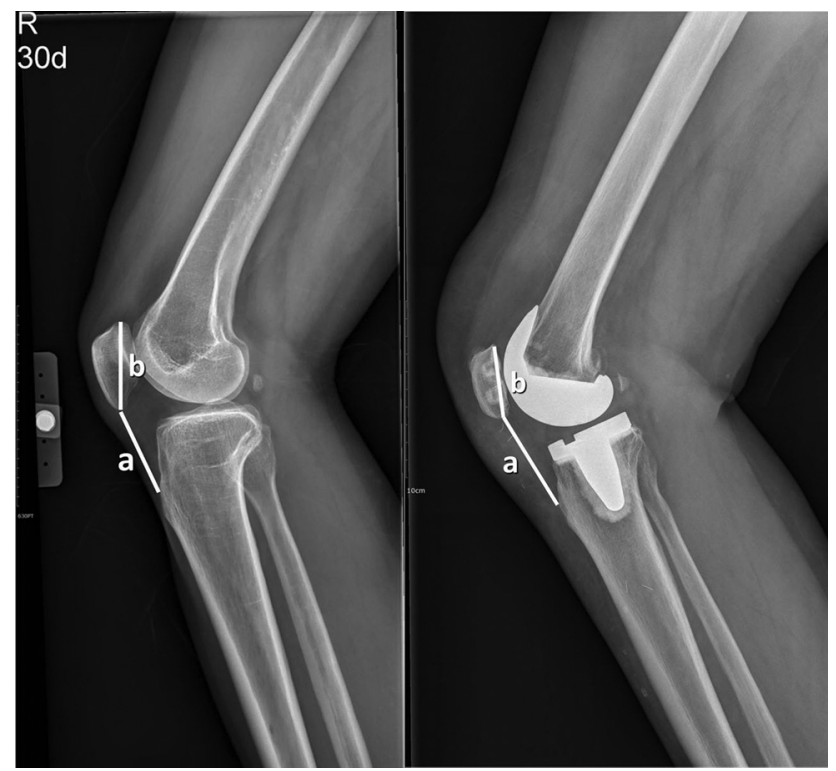

Fig. 3 Measurement of the Insall-Salvati ratio on preoperative and postoperative radiographs (a/b).

length of the patella (b) ( - Fig. 3). The Blackburne-Peel ratio is defined as $\mathbf{a}^{\prime} / \mathbf{b}^{\prime}$; in - Fig. 4. "a" is the perpendicular height of the lower end of the articular surface of the patella from the tibial plateau line, and "b" is the length of the articular surface of the patella. A picture archiving and communication system (INFINITT, Seoul, South Korea) was used for all radiographic measurements.

The flexion contracture and maximal flexion were measured in the supine position using a goniometer preoperatively and at 2 years postoperatively. The clinical status of each knee was rated during each measurement using the visual analog scale pain scale (VAS), Western Ontario and 


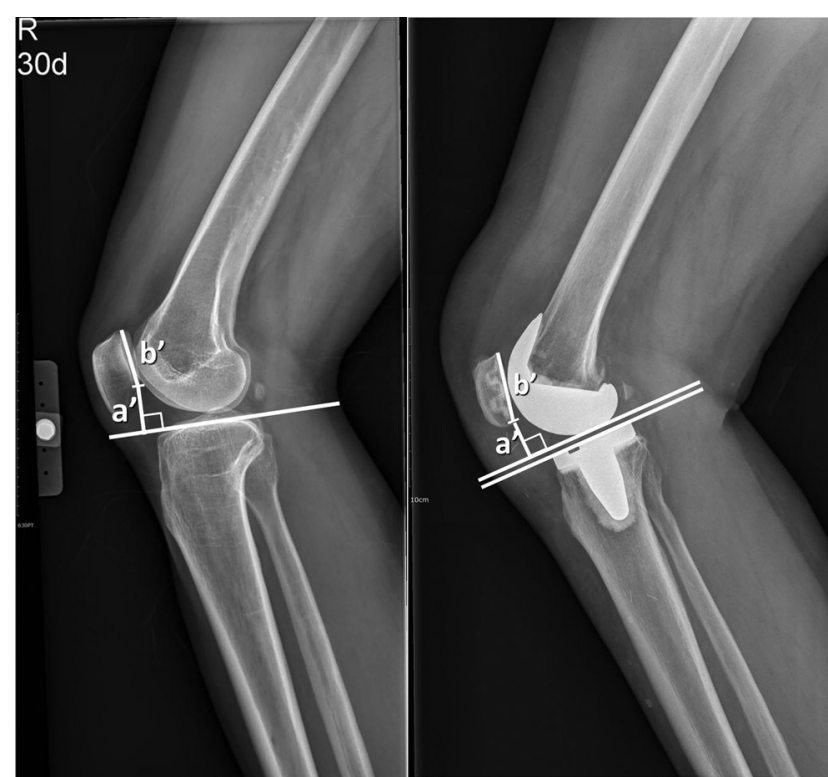

Fig. 4 Measurement of the Blackburne-Peel ratio on preoperative and postoperative radiographs (a'/b').

McMaster University Index (WOMAC), Hospital for Special Surgery Knee Score (HSS), Knee Society (KS) knee and function scores, and Forgotten Joint Score.

\section{Statistical Analysis}

All statistical analyses were performed with SPSS1 22.0 statistical software (SPSS Inc, Chicago, IL). The mean and standard deviation were calculated, and the preoperative and postoperative PTS, Insall-Salvati ratio, Blackburne-Peel ratio, flexion contracture, maximal flexion, ROM, and clinical scores were compared using the paired $t$-test. Patients were then divided into two groups based on the average change in PTS, and the postoperative results were compared between the groups (small PTS change group vs. large PTS change group).

To evaluate affecting factors, the thickness of the PE insert and the postoperative Insall-Salvati ratio, Blackburne-Peel ratio, ROM, VAS, WOMAC, HSS, KS structural and functional scores, and Forgotten Joint Score were subjected to Pearson's correlation analysis to identify the correlation with the individual change in PTS after TKA. In addition, correlation analysis was performed using the detailed questions of each clinical scoring scale. Multivariable regression analysis was then performed to identify factors affecting the clinical scores, which showed statistically significant correlation with the change in PTS (age, BMI, postoperative PTS, change in PTS, polyethylene thickness, postoperative Insall-Salvati ratio, postoperative Blackburne-Peel ratio, and postoperative ROM). A $p$-value of $<0.05$ was considered significant.

To reveal the sample size of this study sufficient for adequate power, the statistical software $G^{*}$ Power (Erdfelder, Faul, Lang \& Buchner, 2007) was used for power analysis. A post hoc power analysis was performed and showed a sufficient sample size for this retrospective study with an $\alpha$ of 0.05 and a power $>0.8$.
The eligibility of measurements was assessed by examining the intrarater and interrater reliability using intraclass correlation coefficients (ICCs). Two trained orthopaedic surgeons working in the knee division of the orthopaedic department evaluated the adequacy of patients' radiograph, especially the true lateral view of the knee joint, and performed two measurements at 2-week intervals. We found good ICCs of the intra- and interobserver reliabilities $(>0.8)$.

\section{Results}

\section{Radiologic and Clinical Results}

The mean PTS changed from $9.6 \pm 3.4$ degrees preoperatively to $2.0 \pm 1.3$ degrees postoperatively $(p<0.001)$, and the mean change in PTS was $7.6 \pm 3.5$ degrees. The mean thickness of the PE insert was $11.7 \pm 1.5 \mathrm{~mm}$ (range, 10-14). The patellar height showed no significant difference after TKA according to the Insall-Salvati ratio (preoperative, $1.04 \pm 0.13$ vs. postoperative, $1.07 \pm 0.14, p=0.147$ ), but a significant difference according to the Blackburne-Peel ratio (preoperative, $0.80 \pm 0.10$ vs. postoperative, $0.67 \pm 0.13, p<0.001$ ). The mean ROM changed from $120.1 \pm 15.4$ degrees preoperatively (range, $70-140$ degrees) to $128.4 \pm 9.3$ degrees postoperatively (range, 90-140 degrees). All clinical scores showed a statistically significant improvement 2 years postoperatively (-Table 2).

\section{Comparison between the Small and Large PTS Change Groups}

Patients were divided into two groups based on an average PTS change of 7.6 degrees: small PTS change group and large PTS change group (-Table 3 ). The mean postoperative change in PTS was significantly larger in the large PTS change group than in the small PTS change group ( $4.9 \pm 2.0$ vs. $10.4 \pm 2.5$ degrees, $p<0.001)$. The mean preoperative PTS was significantly larger in the large PTS change group than in the small PTS change group $(7.2 \pm 2.4$ degrees vs. $12.1 \pm 2.4$ degrees, $p<0.001$ ). Although the mean postoperative PTS was significantly different between the two groups, the postoperative PTS values in the two groups were compatible with our surgical target PTS of 0 to 3 degrees. Therefore, we considered that the difference was not sufficient be clinically significant. Furthermore, pseudo patella baja was more frequently observed in the small PTS change group than in the large PTS change group ( $21.4 \%$ vs. $6.3 \%$, odds ratio $=7.829, p=0.005$ ). There was no significant difference in postoperative ROM and clinical scores between the two groups.

\section{Correlation Analysis for the Effect of the PTS on Postoperative Results}

In terms of correlation analysis between the individual change in PTS after TKA and postoperative results, there was no statistical relationship between the absolute change in PTS and the postoperative ROM (correlation coefficient = $-0.018, p=0.818$ ). Moreover, the change in PTS was not significantly correlated with the polyethylene thickness and patellar height. Regarding correlation with clinical scores, 
Table 2 Radiologic and clinical results

\begin{tabular}{|l|l|l|l|}
\hline & Preoperative & Postoperative & $p$-Value \\
\hline $\begin{array}{l}\text { Posterior tibial slope } \\
\text { (degrees) }\end{array}$ & $9.6 \pm 3.4$ & $2.0 \pm 1.3$ & $<0.001$ \\
\hline $\begin{array}{l}\text { Change of posterior tibial } \\
\text { slope (degrees) }\end{array}$ & $7.6 \pm 3.5$ & \\
\hline Polyethylene thickness (mm) & $11.7 \pm 1.5$ & $1.07 \pm 0.14$ & \\
\hline Insall-Salvati ratio & $1.04 \pm 0.13$ & $0.67 \pm 0.13$ & n.s \\
\hline Blackburne-Peel ratio & $0.80 \pm 0.10$ & $0.3 \pm 1.8$ & $<0.001$ \\
\hline Flexion contracture (degrees) & $8.9 \pm 6.5$ & $128.6 \pm 9.7$ & $<0.001$ \\
\hline Maximal flexion (degrees) & $126.9 \pm 13.0$ & $128.4 \pm 9.3$ & n.s \\
\hline Range of motion (degrees) & $120.1 \pm 15.4$ & $1.4 \pm 2.0$ & $<0.001$ \\
\hline VAS & $7.6 \pm 1.8$ & $8.0 \pm 9.3$ & $<0.001$ \\
\hline WOMAC & $41.9 \pm 14.8$ & $95.9 \pm 4.5$ & $<0.001$ \\
\hline HSS & $69.3 \pm 8.7$ & $96.8 \pm 2.8$ & $<0.001$ \\
\hline KSS Knee & $56.9 \pm 19.3$ & $90.6 \pm 9.2$ & $<0.001$ \\
\cline { 2 - 5 } & Function & $52.9 \pm 14.7$ & $42.7 \pm 24.7$ \\
\hline Forgotten Joint Score & & $<0.001$ \\
\hline
\end{tabular}

Abbreviations: HSS, the Hospital for Special Surgery Knee Score; KSS, Knee Society Score; n.s, not significant; VAS, visual analog scale pain scale; WOMAC, The Western Ontario and McMaster Universities Arthritis Index.

Note: All values are presented as the mean and the standard deviation.

there was no statistical significance between the PTS change and any of the postoperative scoring systems. When correlation analysis was performed using the clinical scoring scale, the absolute change in PTS was found to have a weak positive correlation with WOMAC function, No 10 (difficulty in rising from sitting) (correlation coefficient $=0.342, p=0.041$ ), and moderate positive correlation with the Forgotten Joint Score, No. 6 (awareness when climbing stairs) (correlation coefficient $=0.470, p=0.001$ ) ( - Table 4). In multivariate regression analysis to evaluate factors affecting the WOMAC function No 10 and the Forgotten Joint Score No. 6, which showed a significant correlation with the change in PTS, there was no predictive factor for WOMAC function No. 10. regarding Forgotten Joint Score No. 6, and only BMI was statistically predictive for awareness when climbing stairs after surgery (standardized coefficients $=0.394, p=0.021$ ).

\section{Discussion}

The current study demonstrated that the amount of change in PTS after TKA was not correlated to the postoperative ROM, although it was somewhat related to the ability of a patient to rise from sitting, and their awareness of the affected artificial knee when climbing stairs, 2 years after surgery.

Achieving satisfactory ROM after a TKA is considered an important factor for successful TKA. ${ }^{1,2}$ Surgeons believe that many factors, such as preoperative ROM, BMI, prosthesis design, PTS, and surgical techniques, affect the achievement of maximal ROM after TKA. Several studies with cruciate retaining (CR) type TKAs reported that the postoperative PTS influences the postoperative ROM. ${ }^{3-6}$ Walker and Garg demonstrated that the most important surgical variable related to postoperative maximum flexion was the PTS in the sagittal plane. ${ }^{6}$ Furthermore, Bellemans et al showed that a 1 degree increment of PTS improved the maximal flexion by 1.7 degrees. $^{3}$ In a clinical study with CR TKA, multivariate regression analysis revealed a significant correlation between PTS and postoperative ROM at 12 months follow-up $(p<0.001){ }^{4}$

Several studies have reported that an increased PTS is associated with postoperative flexion angle after PS TKA, as in CR TKA. ${ }^{5,25}$ Indeed, Shi et al reported a positive correlation, in which maximal knee flexion increased by 1.8 degrees when the PTS increased by each degree after PS TKA $(\mathrm{R}=0.681))^{5}$ Furthermore, in a study of 167 patients (209 TKAs), the absolute difference between post- and preoperative PTS was significantly correlated with postoperative flexion $(p<0.001) .{ }^{25}$ However, several studies have denied the relationship between postoperative PTS and ROM. ${ }^{12,13,26}$ Indeed, Oka et al reported that no correlation between the PTS and maximal knee flexion was observed after either PS TKA or CR TKA. ${ }^{13}$ Moreover, Bauer et al reported that there was no correlation between PTS and maximal knee flexion after PS TKA. ${ }^{26}$ Kansara and Markel reported no significant difference in knee flexion after PS TKA between a group of patients with a mean PTS of 1.8 degrees and a group of patients with a mean PTS of 5.5 degrees. ${ }^{12}$ In the current study, the absolute change in PTS was not correlated with postoperative ROM after PS TKA.

The kinematics of knee flexion in PS TKA are different to those in CR TKA, and the effect of the PTS on the postoperative maximum flexion in PS TKA is controversial. ${ }^{13,14,27-29} \mathrm{~A}$ tibial component with increased PTS could lead early campost impingement in full extension. ${ }^{30,31}$ Additionally, 
Table 3 Comparison of postoperative results according to the amount of change in PTS

\begin{tabular}{|l|l|l|l|}
\hline & $\begin{array}{l}\text { Small PTS change group } \\
\text { (PTS change }<\text { 7.6, } \boldsymbol{n}=84 \text { ) }\end{array}$ & $\begin{array}{l}\text { Large PTS change group } \\
\text { (PTS change } \geq 7.6, \boldsymbol{n}=80)\end{array}$ & $p$-Value \\
\hline $\begin{array}{l}\text { Change of PTS } \\
\text { (degrees) }\end{array}$ & $4.9 \pm 2.0$ & $10.4 \pm 2.5$ & $<0.001^{\mathrm{a}}$ \\
\hline $\begin{array}{l}\text { Preoperative PTS } \\
\text { (degrees) }\end{array}$ & $7.2 \pm 2.4$ & $12.1 \pm 2.4$ & $<0.001^{\mathrm{a}}$ \\
\hline $\begin{array}{l}\text { Postoperative PTS } \\
\text { (degrees) }\end{array}$ & $2.4 \pm 1.3$ & $1.7 \pm 1.2$ & $0.001^{\mathrm{a}}$ \\
\hline $\begin{array}{l}\text { Polyethylene thick- } \\
\text { ness (mm) }\end{array}$ & $11.5 \pm 1.6$ & $11.8 \pm 1.5$ & $\mathrm{n} \cdot \mathrm{s}^{\mathrm{a}}$ \\
\hline Insall-Salvati ratio & $1.08 \pm 0.14$ & $1.06 \pm 0.14$ & $\mathrm{n} \cdot \mathrm{s}^{\mathrm{a}}$ \\
\hline Blackburne-Peel ratio & $0.67 \pm 0.14$ & $0.68 \pm 0.12$ & $\mathrm{n} \cdot \mathrm{s}^{\mathrm{a}}$ \\
\hline $\begin{array}{l}\text { Number of pseudo } \\
\text { patella baja }\end{array}$ & $18 / 84(21.4 \%)$ & $5 / 80(6.3 \%)$ & $0.005^{\mathrm{b}}(\mathrm{OR}=7.829)$ \\
\hline $\begin{array}{l}\text { Flexion contracture } \\
\text { (degrees) }\end{array}$ & $0.2 \pm 1.2$ & $0.4 \pm 1.3$ & $\mathrm{n} \cdot \mathrm{s}^{\mathrm{a}}$ \\
\hline $\begin{array}{l}\text { Maximal flexion } \\
\text { (degrees) }\end{array}$ & $128.9 \pm 8.6$ & $128.5 \pm 9.2$ & $\mathrm{n} \cdot \mathrm{s}^{\mathrm{a}}$ \\
\hline $\begin{array}{l}\text { Range of motion } \\
\text { (degrees) }\end{array}$ & $128.7 \pm 9.0$ & $128.2 \pm 9.7$ & $\mathrm{n} \cdot \mathrm{s}^{\mathrm{a}}$ \\
\hline VAS & $1.3 \pm 1.8$ & $1.6 \pm 2.3$ & $\mathrm{n} \cdot \mathrm{s}^{\mathrm{a}}$ \\
\hline WOMAC & $6.6 \pm 9.0$ & $9.3 \pm 9.6$ & $\mathrm{n} \cdot \mathrm{s}^{\mathrm{a}}$ \\
\hline HSS & $95.8 \pm 5.7$ & $96.0 \pm 3.1$ & $\mathrm{n} \cdot \mathrm{s}^{\mathrm{a}}$ \\
\hline KSS & $96.8 \pm 2.8$ & $96.7 \pm 2.9$ & $\mathrm{n} \cdot \mathrm{s}^{\mathrm{a}}$ \\
\hline & $89.9 \pm 10.1$ & $91.2 \pm 8.4$ & $\mathrm{n} \cdot \mathrm{s}^{\mathrm{a}}$ \\
\hline Forgotten Joint Score & $47.4 \pm 24.2$ & $34.4 \pm 24.0$ & $\mathrm{n} \cdot \mathrm{s}^{\mathrm{a}}$ \\
\hline
\end{tabular}

Abbreviations: HSS, the Hospital for Special Surgery Knee Score; KSS, Knee Society Score; n.s, not significant; OR, odds ratios; PTS, posterior tibial slope; VAS, visual analog scale pain scale; WOMAC, The Western Ontario and McMaster Universities Arthritis Index.

The statistical significance was set at $p<0.05$.

a'Derived using the Student's t-test.

${ }^{b}$ Derived using the Pearson chi-squared test.

according to several reports regarding the effect of PCL resection on flexion-extension gaps during TKA, PCL resection increases the flexion gap by $\sim 3$ to $5 \mathrm{~mm}$ compared with the extension gap by $1 \mathrm{~mm} .^{16,32,33}$ Theoretically, the use of a thicker PE insert to fill the flexion gap can block full extension, without additional resection of the distal femur, and worsen the ROM after surgery. ${ }^{34}$ Therefore, efforts to reduce the PTS are necessary to reduce the flexion gap in PS TKA. ${ }^{16}$ Although we investigated whether the thickness of PE insert was related to the change in PTS, the correlation between the two was not statistically significant.

Regarding the effect of postoperative PTS on clinical outcomes, Kansara and Markel found no significant difference in the clinical outcome measured by HSS between a group with 31 cases PS TKAs with a PTS of 0 degrees and a group of 30 PS TKAs with a PTS of 5 degrees. ${ }^{12}$ In our study, patients with a larger change in PTS after PS TKA had more difficulty in "rising from sitting (WOMAC function, No 10)," and were more aware of their knees "when climbing stairs (the Forgotten Joint Score, No. 6)." Indeed, an increase in PTS has been shown to lead to a decrease in maximum quadriceps force and patellofemoral contact force. ${ }^{15}$ On the contrary, a reduction in PTS, due to a larger change in PTS, can impair movement efficiency, which contributes to a reduction in quadriceps force and patellofemoral contact force., 921,35 Decreasing the PTS induces more posterior position of the tibial component and more anterior contact position between the femoral and tibial components. As a result, a reduction in PTS leads to a decrease in the quadriceps lever arm. Therefore, decreasing the PTS may require greater quadriceps force and patellofemoral contact force when engaging in activities that require greater power of the quadriceps muscle, such as rising from sitting, and climbing stairs. However, the current study revealed that the change in PTS after PS TKA had only weak-to-moderate correlation with difficulty in "rising from sitting" and awareness of knees "when climbing stairs." Furthermore, multivariate regression showed no predictive factor affecting the WOMAC function No 10, and only BMI affecting the Forgotten Joint Score No. 6. Therefore, there was a lack of evidence on whether the amount of change in PTS affects postoperative clinical scores, and further study is required to identify the effect of changes in the PTS on postoperative activities, such as rising from sitting, and climbing stairs. 
Table 4 Correlation analysis for the effect of change in posterior tibial slope on postoperative results

\begin{tabular}{|l|l|l|}
\hline & $\begin{array}{l}\text { Correlation } \\
\text { coefficient }\end{array}$ & $p$-Value \\
\hline Polyethylene thickness (mm) & 0.065 & n.s \\
\hline Insall-Salvati ratio & -0.013 & n.s \\
\hline Blackburne-Peel ratio & 0.072 & n.s \\
\hline Range of motion & -0.018 & n.s \\
\hline VAS & -0.064 & n.s \\
\hline WOMAC & 0.067 & n.s \\
\hline HSS & -0.031 & n.s \\
\hline KS knee score & 0.013 & n.s \\
\hline KS function score & 0.007 & n.s \\
\hline FJS & 0.616 & n.s \\
\hline $\begin{array}{l}\text { WOMAC function No. 10: } \\
\text { Difficulty in rising from sitting }\end{array}$ & 0.342 & 0.041 \\
\hline $\begin{array}{l}\text { FJS No.6: Aware when } \\
\text { you are climbing stairs? }\end{array}$ & 0.470 & 0.001 \\
\hline
\end{tabular}

Abbreviations: FJS, Forgotten Joint Score; HSS, the Hospital for Special Surgery Knee Score; KS, Knee Society Sc; n.s, not significant; VAS, visual analog scale pain scale; WOMAC, The Western Ontario and McMaster Universities Arthritis Index.

Note: The statistical significance was set at $p<0.05$.

In this study, tibial resection was performed uniformly within 3 degrees of PTS, which resulted in altered patellar height after TKA compared with the preoperative position. Patellar height affects the efficiency of the quadriceps muscle and the knee extension mechanism. ${ }^{36,37}$ Therefore, the change in patellar height should be evaluated before and after surgery to verify our hypothesis. In the current study, two parameters related to patellar height showed a tendency for pseudo patella baja, in which the patellar tendon is not shortened, but the level of the joint line is elevated. Furthermore, we found that there were more patients with pseudo patella baja in the small change PTS group than in the large change PTS group when we compared the patients divided into two groups based on the average change of PTS. The patellar height had potential variability not only based on the postoperative PTS but also based on other surgical factors including the size and placement of the patellar prosthesis, change in joint line, thickness of PE insert, and position of the tibial implant ${ }^{38}$; these factors are affected by the prosthesis design and the surgical technique. Moreover, patellar resurfacing was performed in most of the included cases (141 cases among 164 cases), which made it difficult to accurately measure the Blackburne-Peel ratio, which is measured relative to the length of the patella articular surface. ${ }^{23,37,39}$ Due to these limitations, it was difficult to clarify whether the effects of the change in patellar height were clinically significant in this study, as noted in previous studies. ${ }^{36,40,41}$ Therefore, we suggest that further studies should be performed to identify the clinical effect of the patellar height and PTS change after PS TKA.
The present study has several limitations. First, this is a retrospective case series, which has potential confounders related to prosthesis design or surgical skill. The findings in this study could be influenced by the built-in PTS degree of PS TKA prosthesis we used in this study. However, as far as surgical confounders, we attempted to minimize the influence by using a single surgeon with considerable skill in TKA to perform all surgeries in a single institution. Second, the evaluation period was only 2 years after surgery; thus, it is necessary to investigate whether the change in PTS affects clinical outcomes at a long-term follow-up after PS TKA. Third, the positive correlation between the change in PTS and clinical scores related to rising from sitting and climbing stairs was relatively weak; thus, we were unable to suggest a cutoff value for the change in PTS that makes the clinical scores worse. However, this study had a strength by the use of detailed questions of each clinical scoring system to conduct the correlation analysis. Fourth, there was a possibility of inaccurate measurement of the PTS depending on the adequacy of the true lateral radiographs and the reliability of the investigators with regard to measurements. However, we excluded all patients whose preoperative and postoperative lateral radiographs could not be obtained in true lateral view, and the ICCs were checked to ensure the reliability of measurements. Finally, we could not suggest the optimal range of PTS after PS TKA because the postoperative PTS values in this study were consistent with our surgical target PTS of 0 to 3 degrees and we could not compare them with the PTS in the outlier range.

\section{Conclusions}

Although proximal tibial resection with a constant target of PTS resulted in individually different changes in the PTS after PS TKA, the amount of change in PTS did not affect the postoperative ROM and clinical scores.

Conflict of Interest

None declared.

\section{References}

1 Matsuda S, Kawahara S, Okazaki K, Tashiro Y, Iwamoto Y. Postoperative alignment and ROM affect patient satisfaction after TKA. Clin Orthop Relat Res 2013;471(01):127-133

2 Van Onsem S, Verstraete M, Dhont S, Zwaenepoel B, Van Der Straeten C, Victor J. Improved walking distance and range of motion predict patient satisfaction after TKA. Knee Surg Sports Traumatol Arthrosc 2018;26(11):3272-3279

3 Bellemans J, Robijns F, Duerinckx J, Banks S, Vandenneucker H. The influence of tibial slope on maximal flexion after total knee arthroplasty. Knee Surg Sports Traumatol Arthrosc 2005;13(03): 193-196

4 Malviya A, Lingard EA, Weir DJ, Deehan DJ. Predicting range of movement after knee replacement: the importance of posterior condylar offset and tibial slope. Knee Surg Sports Traumatol Arthrosc 2009;17(05):491-498

5 ShiX, Shen B, Kang P, Yang J,Zhou Z, Pei F. The effect of posterior tibial slope on knee flexion in posterior-stabilized total knee arthroplasty. Knee Surg Sports Traumatol Arthrosc 2013;21(12):2696-2703

6 Walker PS, Garg A. Range of motion in total knee arthroplasty. A computer analysis. Clin Orthop Relat Res 1991;(262):227-235 
7 Bai B, Baez J, Testa N, Kummer FJ. Effect of posterior cut angle on tibial component loading. J Arthroplasty 2000;15(07):916-920

8 Cinotti G, Sessa P, Ragusa G, et al. Influence of cartilage and menisci on the sagittal slope of the tibial plateaus. Clin Anat 2013; 26(07):883-892

9 Dai Y, Angibaud LD, Jenny JY, Hamad C, Jung A, Cross MB. A softtissue preserving method for evaluating the impact of posterior tibial slope on kinematics during cruciate-retaining total knee arthroplasty: a validation study. Knee 2016;23(06):1074-1082

10 Fujimoto E, Sasashige Y, Tomita T, Iwamoto K, Masuda Y, Hisatome T. Significant effect of the posterior tibial slope on the weightbearing, midflexion in vivo kinematics after cruciate-retaining total knee arthroplasty. J Arthroplasty 2014;29(12):2324-2330

11 Giffin JR, Vogrin TM, Zantop T, Woo SL, Harner CD. Effects of increasing tibial slope on the biomechanics of the knee. Am J Sports Med 2004;32(02):376-382

12 Kansara D, Markel DC. The effect of posterior tibial slope on range of motion after total knee arthroplasty. J Arthroplasty 2006;21 (06):809-813

13 Oka S, Matsumoto T, Muratsu $\mathrm{H}$, et al. The influence of the tibial slope on intra-operative soft tissue balance in cruciate-retaining and posterior-stabilized total knee arthroplasty. Knee Surg Sports Traumatol Arthrosc 2014;22(08):1812-1818

14 Lee HY, Kim SJ, Kang KT, Kim SH, Park KK. The effect of tibial posterior slope on contact force and ligaments stresses in posterior-stabilized total knee arthroplasty-explicit finite element analysis. Knee Surg Relat Res 2012;24(02):91-98

15 Okamoto S, Mizu-uchi H, Okazaki K, Hamai S, Nakahara H, Iwamoto Y. Effect of tibial posterior slope on knee kinematics, quadriceps force, and patellofemoral contact force after posterior-stabilized total knee arthroplasty. J Arthroplasty 2015;30(08):1439-1443

16 Okazaki K, Tashiro Y, Mizu-uchi H, Hamai S, Doi T, Iwamoto Y. Influence of the posterior tibial slope on the flexion gap in total knee arthroplasty. Knee 2014;21(04):806-809

17 de Boer JJ, Blankevoort L, Kingma I, Vorster W. In vitro study of inter-individual variation in posterior slope in the knee joint. Clin Biomech (Bristol, Avon) 2009;24(06):488-492

18 Marouane H, Shirazi-Adl A, Adouni M, Hashemi J. Steeper posterior tibial slope markedly increases ACL force in both active gait and passive knee joint under compression. J Biomech 2014;47 (06):1353-1359

19 Asano H, Hoshino A, Wilton TJ. Soft-tissue tension total knee arthroplasty. J Arthroplasty 2004;19(05):558-561

$20 \mathrm{KimJI}$, Chun SH, Han HS, Lee S, Lee MC. Femoral component rotations in different gap tensions in total knee arthroplasty: a prospective randomized controlled trial. Knee 2017;24(02):439-446

21 Ahmad R, Patel A, Mandalia V, Toms A. Posterior tibial slope: effect on, and interaction with, knee kinematics. JBJS Rev 2016;4(04): e31-e36

22 Yoo JH, Chang CB, Shin KS, Seong SC, Kim TK. Anatomical references to assess the posterior tibial slope in total knee arthroplasty: a comparison of 5 anatomical axes. J Arthroplasty 2008;23(04):586-592

23 Blackburne JS, Peel TE. A new method of measuring patellar height. J Bone Joint Surg Br 1977;59(02):241-242

24 Rogers BA, Thornton-Bott P, Cannon SR, Briggs TWR. Interobserver variation in the measurement of patellar height after total knee arthroplasty. J Bone Joint Surg Br 2006;88(04):484-488
25 Singh G, Tan JH, Sng BY, Awiszus F, Lohmann CH, Nathan SS. Restoring the anatomical tibial slope and limb axis may maximise post-operative flexion in posterior-stabilised total knee replacements. Bone Joint J 2013;95-B(10):1354-1358

26 Bauer T, Biau D, Colmar M, Poux X, Hardy P, Lortat-Jacob A. Influence of posterior condylar offset on knee flexion after cruciate-sacrificing mobile-bearing total knee replacement: a prospective analysis of 410 consecutive cases. Knee 2010;17 (06):375-380

27 Chambers AW, Wood AR, Kosmopoulos V, Sanchez HB, Wagner RA. Effect of posterior tibial slope on flexion and anteriorposterior tibial translation in posterior cruciate-retaining total knee arthroplasty. J Arthroplasty 2016;31(01):103-106

28 Hamai S, Okazaki K, Shimoto T, Nakahara H, Higaki H, Iwamoto Y. Continuous sagittal radiological evaluation of stair-climbing in cruciate-retaining and posterior-stabilized total knee arthroplasties using image-matching techniques. J Arthroplasty 2015;30 (05):864-869

$29 \mathrm{Kim} \mathrm{JH}$. Effect of posterior femoral condylar offset and posterior tibial slope on maximal flexion angle of the knee in posterior cruciate ligament sacrificing total knee arthroplasty. Knee Surg Relat Res 2013;25(02):54-59

30 Callaghan JJ, Rourke MR, Goetz DD, et al. Tibial post impingement in posterior-stabilized total knee arthroplasty. Clin Orthop Relat Res 2002;404:83-88

31 Haas BD. Tibial post impingement in posterior-stabilized total knee arthroplasty. Orthopedics 2006;29(9, Suppl):S83-S85

32 Kadoya Y, Kobayashi A, Komatsu T, Nakagawa S, Yamano Y. Effects of posterior cruciate ligament resection on the tibiofemoral joint gap. Clin Orthop Relat Res 2001;(391):210-217

33 Park SJ, Seon JK, Park JK, Song EK. Effect of PCL on flexionextension gaps and femoral component decision in TKA. Orthopedics 2009;32(10, Suppl):22-25

34 Matziolis G, Loos M, Böhle S, Schwerdt C, Roehner E, Heinecke M. Effect of additional distal femoral resection on flexion deformity in posterior-stabilized total knee arthroplasty. Knee Surg Sports Traumatol Arthrosc 2019

35 Catani F, Fantozzi S, Ensini A, Leardini A, Moschella D, Giannini S. Influence of tibial component posterior slope on in vivo knee kinematics in fixed-bearing total knee arthroplasty. J Orthop Res 2006;24(04):581-587

36 Grelsamer RP. Patella baja after total knee arthroplasty: is it really patella baja? J Arthroplasty 2002;17(01):66-69

37 Prudhon JL, Caton JH, Aslanian T, Verdier R. How is patella height modified after total knee arthroplasty? Int Orthop 2018;42(02): 311-316

38 Phillips CL, Silver DAT, Schranz PJ, Mandalia V. The measurement of patellar height: a review of the methods of imaging. J Bone Joint Surg Br 2010;92(08):1045-1053

39 Cabral F, Sousa-Pinto B, Pinto R, Torres J. Patellar height after total knee arthroplasty: comparison of 3 methods. J Arthroplasty 2017; 32(02):552-557.e2

40 Aguirre-Pastor A, Ortolá DJ, Lizaur-Utrilla A, Rosa MA, Lopez-Prats FA. Is pseudo-patella baja really a serious complication of total knee arthroplasty? J Arthroplasty 2020;35(02):557-562

41 Bugelli G, Ascione F, Cazzella N, et al. Pseudo-patella baja: a minor yet frequent complication of total knee arthroplasty. Knee Surg Sports Traumatol Arthrosc 2018;26(06):1831-1837 